\title{
Innovative Health Technologies Based on Big Data
}

\author{
Serik Smagulov, \\ Viktoriya Smagulova MD
}

\begin{abstract}
This article discusses the possibilities of using big data (Big Data) in innovative technologies in healthcare. We are talking about processing and storing large data which allow making diagnosis more accurately, checking medical data without burdening doctors, and integrating the results of studies performed on different devices into a common system. Big data analysis is also needed to optimize the staffing of healthcare institutions. The author also discusses various healthcare applications for smartphones and other mobile devices that also work on the basis of big data (CareAware Connect, MyChart, Healthtap). Of course, one of the biggest trends in business intelligence remains predictive analytics in healthcare. With examples of using big data in this industry, this article examined the main trends in improving the quality of treatment for patients, especially those suffering from chronic diseases, tracking the general health of the population and reducing the overall costs of medical institutions.
\end{abstract}

Keywords: Big Data, business analytics, mobile applications, electronic health record (HER), telemedicine.

The world of big data represents fertile ground for various IT products. IT solutions can lead to the extension of the life of a particular person and improve the quality of his life. Processing and storing big data allows you to make diagnosis more accurately, to check the medical data without the burden on doctors, and also to integrate the results of studies performed on different devices into a conventional system.

Electronic health records (EHR) are the most common application of big data in medicine [1]. Each patient has his or her digital record, which includes demographic data, medical history, allergies, laboratory test results, etc.

Records are transmitted via secure information systems. Each record consists of one variable file, which means that doctors can make changes over time without paperwork and without the danger of data replication [2].

EHR can also trigger warnings and reminders when a patient has to undergo new laboratory tests or keep track of prescriptions to see if the patient has performed the doctor's prescriptions $[3 ; 4]$.

Although electronic health records are a great idea, many countries have not yet entirely switched to using them. 
Other examples of big data analytics in healthcare have one important function - realtime alerting. For instance, in American hospitals, Clinical Decision Support (CDS) software analyzes medical data on-site, providing advice to physicians when making prescriptive decisions.

Big Data analysis is needed to optimize personnel work. After analyzing the data on visits and determining when a large number of visitors are expected in health care facilities, additional staff can be attracted, which will lead to a reduction in patient waiting times and improving the quality of care.

With regard to patient health data, the latest wearable devices are capable of continuously collecting patient health data and sending this data to the cloud.

For example, if a patient's blood pressure rises alarmingly, the system will send a warning in real time to the doctor, who then takes action to contact the patient and takes measures to reduce pressure.

Another example is Asthmapolis, which began using inhalers with GPS trackers to identify asthma trends at the individual level as well as when looking at large populations. This data is used in conjunction with the CDC data to develop more effective treatment plans for asthmatics.

Of course, predictive analytics in healthcare is one of the most significant trends in business intelligence.

The goal of business intelligence in healthcare is to help physicians make data-based decisions and improve patient care within seconds. This is especially useful in the case of patients with a complex history of the disease, suffering from multiple diseases. New tools will also be able to predict, for example, who is at risk for any diseases, and therefore, additional examinations are recommended.

Of course, as in other industries, there are many obstacles to introducing the latest technologies using Big Data, including incompatible data systems and patient privacy issues. But it is believed that analytics can improve security. In addition, it helps to simplify the processing of insurance claims. 
Big Data can be found in the field of telemedicine. This term refers to the provision of remote clinical services using technology.

Telemedicine is used for the initial consultation and initial diagnosis, remote patient monitoring and medical education for medical professionals [5].

Clinicians use telemedicine to provide personalized treatment plans and prevent hospitalization or re-hospitalization $[6 ; 7]$.

This use of medical data analytics may be associated with the use of predictive analytics, as shown earlier. This allows doctors to predict acute medical events in advance and prevent the patient from worsening. Patients can avoid queues, and doctors do not waste time on unnecessary consultations and paperwork.

Telemedicine also improves access to medical care, since patients can be monitored and counseled at any place and at any time.

Healthcare applications for smartphones and other mobile devices also operate on Big Data [8; 9]. They act as personal assistants.

With this in mind, it is not surprising that all-important mobile device companies integrate tools and features into their devices that help users keep track of their daily physical activity, such as the number of steps taken during the day, heart rate and other vital functions, the amount of calories burned for running, walking or using an elliptical trainer and much more.

Mobile devices and applications are crucial to the health revolution for big data. Big data applications in healthcare send all digital health-related information recorded by mobile devices to cloud servers, directing it to the Big Data database to create reports and support data analysis, including trend recognition.

A person's digital information can be compared and analyzed with thousands of other users, thereby identifying threats, trends, and problems using patterns. This can lead to a complex prognostic model based on data from numerous patients with similar conditions, genetic factors, and lifestyle choices.

Mobile healthcare applications are an integral part of devices designed to access big data medical information. 
Recently, some applications are gaining special popularity. All of them are based on aggregated data and input made to the application.

CareAware Connect is a mobile application that manages clinical communications on a single device for groups that collaborate to better coordinate medical care. Users can view patient data, learn about various health indicators.

MyChart is an application that allows patients to view health data after previous visits to the doctor. Users can see test results, medications, and health status as data provided by a health care provider.

Healthtap is a website and mobile app that serves as an interactive platform for healthcare that initially started as a question and answers service, allowing patients to send health questions to proven doctors. Thanks to it, the company can offer services that include tips on health, telemedicine, and doctors' reviews of specific medicines.

Big data analytics can be used directly in a particular hospital [10]. The medical information panel provides the necessary data. By collecting at one point all the data about each department of the hospital, attendance, its nature, costs incurred, etc., you can get a general picture of the institution, which will be useful for its efficient and smooth operation.

Here you can see the most critical indicators that relate to various aspects: the number of patients who were admitted to the hospital, how long they were and in which department, how much their treatment costs, as well as the average waiting time in emergency departments.

Such a holistic view helps the hospital management to identify potential bottlenecks, identify trends and patterns, and generally assess the situation.

In general, in these examples of using big data in health care, we looked at the main trends in improving the quality of patient care, the general health of the population and the decrease in total costs.

\section{References}

1. Gold R., Cottrell E., Bunce A., Middendorf M., Hollombe C., Cowburn S. Developing electronic health record (EHR) strategies related to health center patients' social determinants of health. J Am Board Fam Med. 2017;30:428-447. 
2. Melanie L. Balestra. Electronic Health Records: Patient Care and Ethical and Legal Implications for Nurse Practitioners // The Journal for Nurse Practitioners February 2017, Volume 13, Issue 2, Pages 105-111.

3. Sue Bowman. Impact of Electronic Health Record Systems on Information Integrity: Quality and Safety Implications // Perspectives in Health Information Management. 2013 Fall; 10(Fall): 1c. Published online 2013 Oct 1.

4. Mandl KD, Khorasani R, Kohane IS: Meaningful use of electronic health records. Health Aff (Millwood) 2012;31.

5. Michael Coakley, Giancarlo Crocetti. Transforming Telemedicine Through Big Data Analytics // semanticscholar.org.

6. Vinayak K. Bairagi. Big Data Analytics in Telemedicine: A Role of Medical Image Compression // Big Data Management pp 123-160

7. Antonino Galletta, Lorenzo Carnevale, Alessia Bramant, Maria Fazio. An Innovative Methodology for Big Data Visualization for Telemedicine // IEEE Transactions on Industrial Informatics. Volume 5. Issue 1.

8. Naoual El aboudi, Laila Benhlima. Big Data Management for Healthcare Systems: Architecture, Requirements, and Implementation // Advances Bioinformatics. 2018; 2018: 4059018. Published online 2018 Jun 21.

9. Blagoj Ristevski, Ming Chen. Big Data Analytics in Medicine and Healthcare // Journal of Integrative Bioinformatics. Volume 15. Issue 3.

10. Viceconti M, Hunter P, Hose R. Big data, big knowledge: big data for personalized healthcare. IEEE J Biomed Health Inform 2015;19:1209-15. 